\title{
Performances Zootechniques, Économiques et Qualité Physique des CEufs Des Poules Soumises À des Régimes Alimentaires Apportant Différentes Concentrations De Tourteau D'amandes de Noix de Cajou (Côte d'Ivoire)
}

\section{Silué Fatogoma Etienne,}

Laboratoire de Nutrition et Pharmacologie, UFR Biosciences, Université Félix Houphouët-Boigny, Abidjan, Côte d'Ivoire

\section{Ouattara Howélé,}

Faculté des Sciences Biologiques,

Université Peleforo Gon Coulibaly, Korhogo, Côte d'Ivoire

\section{Méité Alassane,}

Laboratoire de Nutrition et Pharmacologie, UFR Biosciences,

Université Félix Houphouët-Boigny, Abidjan, Côte d'Ivoire

\section{N'Goran Kouakou David Vincent,}

Laboratoire de Zootechnique,

Institut National Polytechnique Félix Houphouët-Boigny (INP-HB),

Yamoussoukro, Côte d'Ivoire

\section{Véronique Coxam,}

Unité de Nutrition Humaine, INRA

Theix, Clermont-Ferrand, France

Kati-Coulibay Séraphin,

Laboratoire de Nutrition et Pharmacologie, UFR Biosciences,

Université Félix Houphouët-Boigny, Abidjan, Côte d'Ivoire

\section{Résumé}

Cette étude vise à proposer la valorisation des tourteaux de noix de cajou comme source de protéines dans l'alimentation des volailles. Pour ce faire, les paramètres zootechniques, les caractéristiques physiques de l'œuf et la rentabilité financière de la conduite des poules ont été évalués. Le tourteau d'amandes de noix de cajou a été incorporé dans des rations alimentaires équilibrées de 96 poules pondeuses à raison de $0 \%, 10 \%, 15 \%$ et $20 \%$, respectivement pour les régimes codés Rt (témoin), R10, R15 et R20. L'expérimentation a été menée sur huit (8) semaines au cours desquelles chaque poule a reçu quotidiennement en moyenne $125 \mathrm{~g}$ de nourriture. 
Les résultats indiquent que l'incorporation du tourteau d'amandes de noix de cajou dans les régimes induit une diminution du taux moyen de ponte, une légère dégradation du poids de la coquille et de la coloration du jaune de l'œuf. Cependant, il entraine une augmentation significative du gain moyen quotidien des poules et une meilleure rentabilité financière. La consommation alimentaire, l'indice de consommation, les poids moyens de l'œuf, du jaune de l'œuf, du blanc de l'œuf et la composition globale de l'œuf n'ont pas été affectés par l'incorporation du tourteau d'amandes de noix de cajou dans les régimes alimentaires. Les résultats de cette étude révèlent que les tourteaux d'amandes de noix de cajou peuvent être incorporés jusqu'à $20 \%$ dans les régimes alimentaires des poules pondeuses sans compromettre le taux de ponte, les caractéristiques physiques de l'œuf et la rentabilité financière de la conduite des poules. Les tourteaux d'amandes de noix de cajou semblent être une alternative légitime des tourteaux de soja et du maïs dans l'alimentation des volailles.

Mots clés : Côte d'Ivoire, Amandes De Noix De Cajou, Tourteau, Poule Pondeuse, Euf 


\title{
Zootechnical, Economic and Eggs Physical Quality Performances from Chickens Subjected to Diets Providing Different Concentrations of Cashew Nut Meal (Côte d'Ivoire)
}

\author{
Silué Fatogoma Etienne, \\ Laboratoire de Nutrition et Pharmacologie, UFR Biosciences, \\ Université Félix Houphouët-Boigny, Abidjan, Côte d'Ivoire \\ Ouattara Howélé, \\ Faculté des Sciences Biologiques, \\ Université Peleforo Gon Coulibaly, Korhogo, Côte d'Ivoire \\ Méité Alassane, \\ Laboratoire de Nutrition et Pharmacologie, UFR Biosciences, \\ Université Félix Houphouët-Boigny, Abidjan, Côte d'Ivoire \\ N'Goran Kouakou David Vincent, \\ Laboratoire de Zootechnique, \\ Institut National Polytechnique Félix Houphouët-Boigny (INP-HB), \\ Yamoussoukro, Côte d'Ivoire \\ Véronique Coxam, \\ Unité de Nutrition Humaine, INRA \\ Theix, Clermont-Ferrand, France \\ Kati-Coulibay Séraphin, \\ Laboratoire de Nutrition et Pharmacologie, UFR Biosciences, \\ Université Félix Houphouët-Boigny, Abidjan, Côte d'Ivoire
}

\begin{abstract}
This study aims to propose the promotion of cashew meal as a source of protein in poultry feed. To do this, the zootechnical parameters, the physical characteristics of the egg and the financial profitability of the management of the hens were evaluated. The cashew nut meal was incorporated into balanced feed rations of 96 laying hens at $0 \%, 10 \%, 15 \%$ and $20 \%$ respectively for the coded diets Rt (control), R10, R15 and R20. The experiment was carried out over eight (8) weeks during which each hen received an average of $125 \mathrm{~g}$ of food daily. The results indicate that the incorporation of the cashew nut kernel meal in the diets induces a reduction in the average rate of spawning, a slight deterioration in the weight of the shell and the coloration of the yolk of the egg. However, it results in a significant increase in the average daily gain of
\end{abstract}


the hens and better financial profitability. Food consumption, consumption index, average egg weights, egg yolk, egg white, and overall egg composition were not affected by the incorporation of cashew nut kernel meal into diets. The results of this study reveal that cashew nut can be incorporated up to $20 \%$ into the diets of laying hens without compromising the egg-laying rate, the physical characteristics of the egg and the financial profitability of the management of hens. The cashew almond meal could to be a legitimate alternative to soybean meal and corn in the poultry feed.

Keywords: Ivory Coast, Cashew Nuts, Kernel Meal, Layer Hen, Egg

\section{Introduction}

Les nutritionnistes européens s'éloignent régulièrement du modèle "céréale-soja" en valorisant divers sous-produits et protéagineux tandis que leurs confrères du Sud restent paradoxalement plus conservateurs. Des masses croissantes de céréales et de soja sont importées du Nord, entrainant ainsi d'importantes sorties de devises (Picard et al., 1993). En effet, dans la plupart des pays en développement, des quantités conséquentes de sous-produits et de résidus agricoles sont générées chaque année. Ces sous-produits qui ont tendance à s'accumuler pourraient pourtant être valorisés dans l'alimentation animale (Jayathilakan et al., 2012).

Dans ces conditions, les efforts de recherche doivent être axés sur l'évaluation de la qualité et des potentialités de ces produits et résidus agricoles non conventionnels et localement disponibles. Ainsi, en Côte d'Ivoire, premier producteur mondial de noix de cajou, les amandes de noix de cajou pourraient être une alternative intéressante au tourteau de soja importé à des coûts élevés (Atteh et Ologbenla, 1993, Aduku, 1993 ; Odunsi, 2002). En Côte d'Ivoire, peu de données scientifiques relatives à la valorisation des tourteaux d'amandes de noix de cajou dans l'alimentation animale, en particulier chez les poules pondeuses, sont disponibles. Ce travail a été réalisé pour essayer de valorisation le tourteau d'amandes de noix de cajou.

L'objectif de cette étude est donc d'évaluer l'effet suscité par l'incorporation de tourteau d'amandes de noix de cajou dans les rations alimentaires de poules pondeuses sur les performances zootechniques, économiques et la qualité physique des œufs.

\section{Matériel et méthodes d'étude}

\section{Matériel}

\section{Infrastructures d'élevage}

Le poulailler expérimental de l'Ecole de Spécialisation en Elevage et Métiers de la Viande de Bingerville (ESEMV-B) a été choisi pour conduire 
l'essai. D'une superficie de $60 \mathrm{~m} 2$, c'est une structure ouverte avec un grillage reposant sur un muret de $30 \mathrm{~cm}$ de haut. Le poulailler a été compartimenté en 24 loges de $1 \mathrm{~m} \mathrm{x} \mathrm{1,35} \mathrm{m} \mathrm{soit} \mathrm{1,35} \mathrm{m2} \mathrm{chacune} \mathrm{avec} \mathrm{un} \mathrm{sol} \mathrm{couvert} \mathrm{de} \mathrm{litière}$ pour installer les poules dans de meilleures conditions d'élevage.

\section{Matériel animal}

Quatre-vingt-seize (96) poulettes, âgées de 20 semaines, de souche LOHMAN-BROWN, d'un poids moyen de $1600 \mathrm{~g}$, ont été utilisées dans cette étude. Celles-ci ont été élevées à la ferme expérimentale de l'ESEMV-B.

\section{Matériel alimentaire}

Les aliments expérimentaux étaient composés de plusieurs intrants que sont le tourteau de soja importé, le maïs jaune, les tourteaux de noix de cajou et de coton, le son de blé, la farine basse de riz, la farine de poisson, le coquillage, le prémix TNH 1,25\%, le Sépiolite et le sel issus du commerce. Les amandes déclassées de noix de cajou utilisées pour produire le tourteau provenaient des usines SOBERY à Bouaké et CAJU INDUSTRIE de Kolia, respectivement au Centre et au nord de la Côte d'Ivoire. Les aliments expérimentaux contenaient un taux croissant de tourteau d'amandes de noix de cajou (Rt avec $0 \%, \mathrm{R} 10$ avec $10 \%$, R15 avec $15 \%$, et R20 avec $20 \%$ ). Ils ont été formulés dans l'objectif d'être isoénergétiques et isoprotéiques. Les contraintes minimales d'optimisation en acides aminés digestibles étaient de $0,76 \%$ pour la lysine, $0,47 \%$ pour la méthionine. En conséquence de cette formulation, le tourteau d'amandes de noix de cajou n'est pas la seule matière première variable dans les formules (Tableau I).

Les aliments expérimentaux, fabriqués à l'atelier expérimental de l'ESEMVB, ont été broyés à l'aide d'un broyeur à marteaux et mélangés dans des conditions identiques. Des analyses physico-chimiques réalisées au Laboratoire de Zootechnique de l'Institut National Polytechnique Felix Houphouët Boigny (INP-HB) sur les échantillons d'aliments ont permis de valider leur conformité analytique.

\begin{tabular}{lcccc}
\hline \multicolumn{4}{c}{ Régimes alimentaires } & \\
\hline Ingrédients (en Kg) & $\mathbf{R t}$ & $\mathbf{R 1 0}$ & $\mathbf{R 1 5}$ & $\mathbf{R 2 0}$ \\
\hline Maïs & 0,6455 & 0,5100 & 0,4434 & 0,3655 \\
Son de blé & 0,0000 & 0,0300 & 0,0600 & 0,0700 \\
Farine basse de riz & 0,0000 & 0,0500 & 0,0700 & 0,1100 \\
T Soja 44 & 0,2060 & 0,1296 & 0,0605 & 0,0503 \\
T Cajou & 0,0000 & 0,1000 & 0,1500 & 0,2000 \\
T Coton & 0,004 & 0,0279 & 0,0500 & 0,0500 \\
Farine Poisson & 0,0400 & 0,0400 & 0,0537 & 0,0417 \\
Coquille huitres & 0,0880 & 0,0880 & 0,0880 & 0,0880
\end{tabular}




\begin{tabular}{lcccc} 
Prémix TNH Ponte 1,25 \% RCI & 0,0125 & 0,0125 & 0,0125 & 0,0125 \\
Sel (NaCl) & 0,0020 & 0,0020 & 0,0020 & 0,0020 \\
Sépiolite & 0,0020 & 0,0100 & 0,0100 & 0,0100 \\
\hline \multicolumn{1}{c}{ TOTAL (Kg) } & $\mathbf{1}$ & $\mathbf{1}$ & $\mathbf{1}$ & $\mathbf{1}$ \\
\hline Valeurs calculées & & & & \\
Matière Sèche (MS) en \% & 88 & 88 & 88 & 89 \\
Energie métabolisable (kcal / kg) & 2756 & 2756 & 2756 & 2756 \\
Protéine Brute (\%) & 17,28 & 17,28 & 17,28 & 17,28 \\
Matière grasse (\%) & 3,05 & 5,05 & 6,13 & 7,13 \\
Cellulose brute (\%) & 2,79 & 3,95 & 4,53 & 5,30 \\
Calcium (\%) & 3,35 & 3,37 & 3,46 & 3,41 \\
Phosphore (\%) & 0,17 & 0,22 & 0,29 & 0,29 \\
\hline Coût (FCFA/Kg d'aliment) & 218 & 206 & 195 & 189 \\
\hline
\end{tabular}

Tableau I : Composition chimique des différents régimes alimentaires

\section{Méthodes}

\section{Production du tourteau d'amandes de noix de cajou}

Le tourteau d'amandes de noix de cajou, riches en éléments nutritifs, est issu de la pression des résidus des amandes de noix de cajou. Il a été produit à la ferme expérimentale de l'ESEMV-B selon la technique utilisée par Kouadio et al., (2016) pour le tourteau d'hévéa. Les amandes de noix de cajou sèches ont été broyées avant d'être mélangées de manière homogène à l'eau à raison de deux litres d'eau pour $10 \mathrm{~kg}$ d'amandes broyées. L'homogénat a été placé dans une cuve et couvert par une bâche, puis séché pendant deux heures au soleil. Par la suite, l'homogénat séché a été pressé à l'aide d'une presse artisanale «presse à manioc ». Après le recueil de 3 litres d'extrait lipidique pour $10 \mathrm{~kg}$ d'homogénat, le bloc de tourteau résultant a été concassé manuellement puis les mottes ont été séchées à nouveau. Le tourteau d'amandes de noix de cajou obtenu et qui a également fait l'objet d'analyses chimiques présentait des teneurs en protéine et lipide de $25,75 \%$ et $21,98 \%$ respectivement.

\section{Dispositif expérimental et conduite de l'essai}

Quatre-vingt-seize (96) poules âgées de 21 semaines ont été distribuées dans un dispositif complètement aléatoire avec quatre régimes dénommés Rt, R10, R15 et R20 correspondant respectivement au taux d'incorporation du tourteau de noix de cajou à $0 \%, 10 \%, 15 \%$ et $20 \%$. Chaque régime expérimental a été répété sur six (6) groupes contenant chacun quatre (4) poules logées dans un bâtiment sur un sol recouvert d'une litière de copeau de bois à raison de 6 kg/m2 (Mehmet et al, 2012 ; ITAVI, 1980). 
En prélude à cette expérimentation, le poulailler a été désinfecté puis laissé fermer durant deux semaines (vide sanitaire) pour qu'il sèche complètement avant le démarrage de l'essai. L'étude a duré dix (10) semaines réparties en deux phases qui sont l'adaptation en deux (2) semaines et l'expérimentation proprement dite en huit (8) semaines.

Les poules ont été nourries avec l'aliment témoin pendant la phase d'adaptation. A la suite de cette période, l'expérimentation a été menée sur huit (8) semaines au cours desquelles chaque oiseau a reçu quotidiennement $125 \mathrm{~g}$ d'aliment et de l'eau à volonté (Lessire et al., 2005 ; Kouakou et al., 2015).

Les poules étaient pesées en début d'expérimentation et chaque fin de semaine. Également, les restes d'aliments distribués, les œufs pondus dans chaque lot ont été récoltés et pesés. A la fin de l'expérimentation, les paramètres zootechniques pour chaque lot tels que le taux de mortalité, la consommation moyenne de nourriture, l'indice de consommation, la variation pondérale et le taux de ponte ont été évalués (Larid, 2012). L'étude de la qualité des œufs a porté sur les caractéristiques physiques (poids moyen des œufs, des coquilles, du jaune, du blanc, la coloration et la composition globale de l'œuf) (Shakeel, 2010).

L'évaluation économique a consisté à l'établissement des coûts de production, du produit d'exploitation, de la marge bénéficiaire brute et de la rentabilité financière pour chaque régime alimentaire (Perrin et al., 1979)..

\section{Analyses statistiques}

Les résultats obtenus au cours cette étude ont été analysés grâce au logiciel Statistica Version 7.1. Les valeurs moyennes par régime issues des critères d'études ont été soumises à une analyse de variance (ANOVA), suivie d'une comparaison de moyenne selon le test de Newman-Keuls au seuil de signification de $5 \%$. Les calculs numériques et la construction des graphiques ont été effectués avec le logiciel Excel.

\section{Résultats}

\section{Effet de la consommation du tourteau de noix de cajou sur les paramètres zootechniques \\ Taux de mortalité}

Les poules nourries avec les différents régimes alimentaires constitués avaient un aspect globalement normal. Aucune mortalité n'a été relevée au cours de l'expérimentation, quel que soit le régime. Cependant, quelques cas de diarrhées ont été constatés de façon générale (Tableau II). 


\section{Consommation moyenne alimentaire par semaine}

Les poules nourries avec le régime témoin à base de tourteau de soja (Rt), ont consommé chaque semaine $876,66 \pm 20,59 \mathrm{~g}$ d'aliment formulé. Les niveaux de consommation hebdomadaires étaient de 861,33 $\pm 25,40 \mathrm{~g}, 893,73$ $\pm 12,65 \mathrm{~g}$ et $880,98 \pm 10,22 \mathrm{~g}$, respectivement pour les régimes $\mathrm{R} 10$, R15 et R20 (Tableau II). L'analyse statistique ne révèle pas de différence significative $(p \geq 0,05)$ entre la consommation alimentaire des poules recevant l'alimentation témoin (Rt) et celle des poules nourries avec les régimes R10, R15 et R20.

\section{Gain moyen quotidien}

Les gains moyens quotidiens (GMQ) enregistrés étaient de 1,78 $\pm 0,42$ $\mathrm{g} / \mathrm{j}, 2,97 \pm 0,33 \mathrm{~g} / \mathrm{j}, 2,62 \pm 0,50 \mathrm{~g} / \mathrm{j}$ et $3,62 \pm 0,13 \mathrm{~g}$, respectivement pour les poules consommant les régimes Rt, R10, R15 et R20. Le GMQ le plus élevé a été obtenu avec le régime alimentaire R20 (3,62 \pm 0,62 g) (Tableau II). Après deux semaines d'alimentation, les poules consommant le régime alimentaire $\mathrm{R} 20$ ont eu une meilleure croissance que celles du groupe témoin ( $\mathrm{Rt}$ ) et celles recevant les régimes $\mathrm{R} 10$ et $\mathrm{R} 15 \%$ (Figure 1). L'analyse statistique a révélé que le GMQ des poules soumises au régime témoin $(\mathrm{Rt})$ était significativement inférieur $(\mathrm{p}<0,05)$ à celui des poules du lot R20.

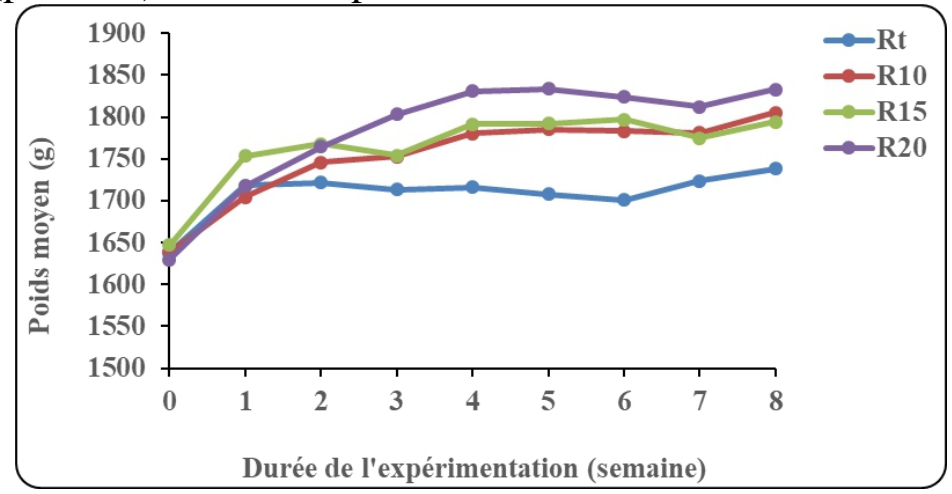

Figure 1 : Courbe de croissance des poules soumises aux divers régimes expérimentaux Chaque valeur est la moyenne \pm écart type de 6 lots de 4 poules. Rt, R10, R15 et R20 sont les régimes alimentaires contenant respectivement $0 \%, 10 \%, 15 \%$ et $20 \%$ de tourteau d'amande de noix de cajou.

\section{Indice de consommation}

L'indice de consommation du régime témoin Rt est de 3,23 $\pm 0,37$. Les indices de consommation enregistrés chez les régimes avec du tourteau d'amandes de noix de cajou sont $3,53 \pm 0,33 ; 3,62 \pm 0,24$ et 4,02 $\pm 0,20$ respectivement pour R10, R15 et R20 (Tableau II).

Au plan statistique, les indices de consommation des différents régimes ne présentent aucune différence significativement $(\mathrm{P} \geq 0,05)$ entre eux cependant, 
il existe une tendance d'un indice de consommation d'un niveau plus important dans le cas des régimes étudiés.

\section{Taux moyen de ponte}

Les taux moyens de ponte enregistrés sur la durée de l'expérimentation

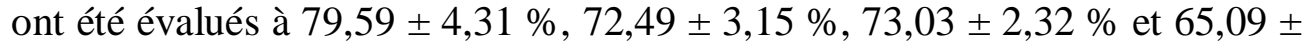
$2,06 \%$ respectivement pour les régimes alimentaires Rt, R10, R15 et R20. La baisse de ponte par rapport au régime témoin est de 8,92 \%, 8,24 \%, 18,22 \% respectivement pour les régimes alimentaires R10, R15 et R20. Les analyses statistiques ont révélé que le taux de ponte moyen chez les poules nourries au régime R20 est significativement faible $(\mathrm{p}<0,05)$ par rapport au taux observé chez les poules nourries au régime témoin ( $\mathrm{Rt}$ ) (Tableau II).

Tableau II : Effet de l'incorporation du tourteau d'amandes de noix de cajou sur les paramètres zootechniques des poules pondeuses

\begin{tabular}{lcccc}
\hline \multirow{2}{*}{ Paramètres mesurés } & Rt & \multicolumn{2}{c}{ Régimes alimentaires } \\
& 0 & $\mathbf{R 1 0}$ & $\mathbf{R 1 5}$ & $\mathbf{R 2 0}$ \\
\hline Taux de mortalité $(\%)$ & $876,66 \pm$ & $861,33 \pm$ & $893,73 \pm$ & $880,98 \pm$ \\
Consommation alimentaire & $20,59^{\mathrm{a}}$ & $25,40^{\mathrm{a}}$ & $12,65^{\mathrm{a}}$ & $10,22^{\mathrm{a}}$ \\
par semaine & $1,78 \pm 0,42^{\mathrm{a}}$ & $2,97 \pm 0,33^{\mathrm{ab}}$ & $2,62 \pm 0,50^{\mathrm{ab}}$ & $3,62 \pm 0,13^{\mathrm{b}}$ \\
Gain moyen quotidien $(\mathrm{g} / \mathrm{j})$ & $3,23 \pm 0,37^{\mathrm{a}}$ & $3,53 \pm 0,33^{\mathrm{a}}$ & $3,62 \pm 0,24^{\mathrm{a}}$ & $4,02 \pm 0,20^{\mathrm{a}}$ \\
Indice de consommation & $79,59 \pm 4,31^{\mathrm{b}}$ & $72,49 \pm 3,1^{\mathrm{b}}$ & $73,03 \pm 2,32^{\mathrm{b}}$ & $65,09 \pm 2,0^{\mathrm{a}}$ \\
Taux moyen de ponte $(\%)$ & & & & \\
Baisse de ponte par rapport & - & 8,92 & 8,24 & 18,22 \\
à Rt $(\%)$ & & & & \\
\hline
\end{tabular}

$a, b$ Il n'y a aucune différence significative $(p \geq 0,05)$ entre deux moyennes \pm écart-type (sur une même ligne).

Effet de la consommation du tourteau de noix de cajou sur les caractéristiques physiques de l'œuf

Poids moyen des oufs

Le poids moyen des œufs du régime témoin est de $58,18 \pm 0,77 \mathrm{~g}$. Les poules des régimes R10, R15 et R20 ont des poids moyens d'œufs respectivement de 57,28 $\pm 0,81 \mathrm{~g}, 56,93 \pm 0,55 \mathrm{~g}$ et 56,21 $\pm 0,91 \mathrm{~g}$. L'analyse statistique indique qu'il n'y a pas de différence significative $(\mathrm{p} \geq 0,05)$ entre les différents lots expérimentaux (Tableau III).

\section{Poids moyen du jaune des œufs}

Le poids moyen du jaune des œufs des poules du régime témoin $\mathrm{Rt}$ était de 13,39 $\pm 0,15 \mathrm{~g}$. Les poids moyens de jaune d'œuf calculés chez les poules ayant consommé les régimes R10, R15 et R20 étaient respectivement de $12,65 \pm 0,22 \mathrm{~g}, 12,90 \pm 0,22 \mathrm{~g}$ et 13,058 $\pm 0,20 \mathrm{~g}$ (Tableau III). Ces 
différentes valeurs ne présentent aucune différence significative $(p \geq 0,05)$ entre elles.

\section{Poids moyen du blanc des œufs}

Les poules ayant consommé les régimes R10, R15 et R20 ont des poids moyens de blanc des œufs respectivement de 38,09 $\pm 0,67 \mathrm{~g}, 37,61 \pm 0,66 \mathrm{~g}$ et $37,80 \pm 0,63 \mathrm{~g}$ contre $38,18 \pm 0,87 \mathrm{~g}$ pour celles soumises au régime témoin Rt. Ces différentes valeurs ne sont pas significativement différentes $(p \geq 0,05)$ (Tableau III).

Tableau III : Effet de l'incorporation du tourteau de noix de cajou sur les caractéristiques physiques des œufs des poules pondeuses

\begin{tabular}{|c|c|c|c|c|}
\hline \multirow{2}{*}{ Paramètres mesurés } & \multicolumn{4}{|c|}{ Régimes alimentaires } \\
\hline & $\mathbf{R t}$ & R10 & R15 & $\mathbf{R 2 0}$ \\
\hline Poids moyen de l'œuf (g) & $58,18 \pm 0,77^{a}$ & $57,28 \pm 0,81^{\mathrm{a}}$ & $56,93 \pm 0,55^{\mathrm{a}}$ & $56,21 \pm 0,91^{\mathrm{a}}$ \\
\hline $\begin{array}{l}\text { Poids moyen du jaune de } \\
\text { l'œuf }(\mathrm{g})\end{array}$ & $13,39 \pm 0,15^{\mathrm{a}}$ & $12,65 \pm 0,22^{\mathrm{a}}$ & $12,90 \pm 0,22^{\mathrm{a}}$ & $13,058 \pm 0,20^{\mathrm{a}}$ \\
\hline $\begin{array}{l}\text { Poids moyen du blanc de } \\
\text { l'œuf }(\mathrm{g})\end{array}$ & $38,18 \pm 0,87^{a}$ & $38,09 \pm 0,67^{\mathrm{a}}$ & $37,61 \pm 0,66^{\mathrm{a}}$ & $37,80 \pm 0,63^{a}$ \\
\hline $\begin{array}{l}\text { Poids moyen de la coquille de } \\
\text { l'œuf (g) }\end{array}$ & $7,13 \pm 0,13^{\mathrm{b}}$ & $6,85 \pm 0,28^{a b}$ & $6,75 \pm 0,12^{a b}$ & $6,21 \pm 0,12^{\mathrm{a}}$ \\
\hline$\%$ du jaune de l'œuf & $23,05 \pm 0,38^{\mathrm{a}}$ & $22,11 \pm 0,51^{\mathrm{a}}$ & $22,67 \pm 0,09^{\mathrm{a}}$ & $23,27 \pm 0,56^{\mathrm{a}}$ \\
\hline$\%$ du blanc de l'œuf & $65,61 \pm 1,06^{\mathrm{a}}$ & $66,50 \pm 0,68^{a}$ & $66,05 \pm 0,75^{\mathrm{a}}$ & $67,26 \pm 0,52^{\mathrm{a}}$ \\
\hline$\%$ de la coquille de l'œuf & $12,24 \pm 0,38^{a}$ & $11,96 \pm 0,46^{\mathrm{a}}$ & $11,88 \pm 0,28^{\mathrm{a}}$ & $11,07 \pm 0,35^{\mathrm{a}}$ \\
\hline $\begin{array}{l}\text { Coloration du jaune de l'œuf } \\
\text { (ER) }\end{array}$ & $6,33 \pm 0,33^{\mathrm{b}}$ & $5,00 \pm 0,00^{\mathrm{a}}$ & $4,67 \pm 0,33^{\mathrm{a}}$ & $4,67 \pm 0,21^{\mathrm{a}}$ \\
\hline
\end{tabular}

a, b Il n'y a aucune différence significative $(p \geq 0,05)$ a, b Il n'y a aucune différence significative $(\mathrm{p} \geq 0,05)$ entre deux moyennes \pm écart-type (sur une même ligne).

ER : Eventail de Roch.

\section{Poids moyen des coquilles des oufs}

$\mathrm{Au}$ terme de l'expérimentation, les poids moyens des coquilles des œufs des poules ayant consommé les différents régimes se situaient entre 6,21 $\pm 0,12 \mathrm{~g}$ et $7,13 \pm 0,29 \mathrm{~g}$. Le poids moyen des coquilles le plus élevé $(7,13 \pm$ $0,13 \mathrm{~g}$ ) était observé chez celles du groupe témoin Rt et celui le plus faible chez les poules ayant reçu le plus fort taux de tourteaux d'amandes de noix de cajou R20 (6,21 $\pm 0,12 \mathrm{~g})$. Les autres poules soumises aux régimes $\mathrm{R} 10$ et $\mathrm{R} 15$ avaient des poids moyens des coquilles des œufs respectivement de 6,85 \pm $0,28 \mathrm{~g}$ et $6,75 \pm 0,12 \mathrm{~g}$ (Tableau III). Les poids moyens des coquilles des œufs des poules nourries avec le régime témoin Rt étaient donc statistiquement plus élevés $(\mathrm{p}<0,05)$ que celles nourries au régime R20. 


\section{Composition globale de l'œuf}

La composition globale de l'œuf indique que les proportions varient de $22,11 \pm 0,51 \%$ à $23,27 \pm 0,56 \%$ pour le jaune d'œuf, de $65,61 \pm 1,06 \%$ à $67,26 \pm 0,52 \%$ pour le blanc d'œuf et de $11,07 \pm 0,35 \%$ à $12,24 \pm 0,38 \%$ pour la coquille (Tableau III). Il n'y a pas de différence significative $(p \geq 0,05)$ entre les pourcentages moyens des composantes des œufs des différents régimes.

\section{Coloration du jaune de l'œuf}

La coloration moyenne du jaune d'œuf des régimes, mesurée sur une échelle de 1 à 15 de l'éventail de couleur DSM, se situait entre 4,67 $\pm 0,21$ et $6,33 \pm 0,33$ (Tableau III). Une coloration moyenne du jaune d'œuf de 6,33 \pm 0,33 était obtenue dans le cas de consommation du régime témoin $\mathrm{Rt}$, tandis que chez les autres poules sous régimes R10, R15 et R20 les colorations moyennes du jaune d'œuf étaient respectivement de 5,00 $\pm 0,00 ; 4,67 \pm 0,33$ et 4,67 $\pm 0,21$. Les colorations moyennes du jaune des œufs des poules nourries avec le régime témoin Rt étaient statistiquement plus élevées ( $\mathrm{p}<$ $0,05)$ que celles nourries avec les régimes R20, R15 et R10.

\section{Effet de la consommation du tourteau d'amandes de noix de cajou sur la rentabilité financière des lots de poules}

Le tableau IV présente la marge bénéficiaire et la rentabilité financière de la production des poules des différents groupes de poules en fonction des régimes.

Les marges bénéficiaires les plus importantes ont été obtenues au niveau des poules des régimes R15 et R10 avec les montants respectifs de 2 447429 FCFA et 2426352 FCFA. Le traitement qui a engendré la plus faible marge bénéficiaire avec un montant de 2320402 FCFA, a été le régime $\mathrm{R} 20$. Le témoin $\mathrm{Rt}$ a aussi dégagé la marge bénéficiaire la plus faible après R20 avec un montant de 2421249 FCFA.

La partition des régimes en fonction de leur rentabilité financière par la classification hiérarchique (dendrogramme) a permis d'isoler deux groupes de poules avec les quatre régimes alimentaires.

La première classe est constituée par les régimes R10 et R15 qui ont induit la meilleure rentabilité financière respectivement de 0,67 et 0,68. La seconde classe est représentée par les régimes Rt et R20 et constitue le groupe avec les plus faibles rentabilités respectivement de 0,65 et 0,66. Il est à noter que bien que les rentabilités des régimes $\mathrm{Rt}$ et $\mathrm{R} 20$ soient similaires, le régime témoin reste celui présentant la plus faible rentabilité. Concernant la rentabilité financière, pour les quatre groupes de poules, le ratiobénéfice/coût a été supérieur à 0,5 pour l'ensemble des régimes alimentaires. 
Tableau IV: Marge brute et rentabilité financière de la production des poules sur la période en fonction des régimes

\begin{tabular}{|c|c|c|c|c|}
\hline \multirow{2}{*}{ Rubriques } & \multicolumn{4}{|c|}{ Régimes alimentaires } \\
\hline & $\mathbf{R t}$ & R10 & R15 & $\mathbf{R 2 0}$ \\
\hline Coût de l'aliment (FCFA/Kg) & 218 & 206 & 195 & 189 \\
\hline Coût de production (FCFA) & 3728895 & 3619472 & 3594219 & 3532042 \\
\hline Recettes (FCFA) & 6150144 & 6045824 & 6041648 & 5852444 \\
\hline Marge brute (FCFA) & 2421249 & 2426352 & 2447429 & 2320402 \\
\hline Rentabilité financière & 0,65 & 0,67 & 0,68 & 0,66 \\
\hline
\end{tabular}

\section{Discussion}

La croissance ou la production d'un animal s'apprécie par son gain de poids et ses performances au cours de son cycle de vie.

Au cours de cette étude, la mortalité a été nulle pour les poules soumises aux différents régimes expérimentaux (R10, R15 et R20), ce qui indique que le tourteau d'amandes de noix de cajou ne contiendrait pas de facteurs antinutritionnels létaux pour la volaille. Selon certains travaux, les tourteaux contiennent une quantité intéressante de substances nutritionnelles excellentes telles que les acides aminés pour la viabilité des poules aussi bien en cage qu'au sol. Ceci est particulièrement vrai dans les pays d'Europe de l'Est où les taux de mortalité restent faibles alors que les tourteaux de tournesol sont utilisés à des teneurs extrêmement élevées (>20\%) dans l'alimentation de volaille (Hartini et al., 2003 ; Joly et Loiselet, 2005).

Concernant la consommation alimentaire, les différences enregistrées entre les poules soumises aux différents régimes, ne sont pas significatives attestant que l'appétence du tourteau d'amandes de noix de cajou est comparable à celui du régime témoin, quelle que soit la concentration à laquelle il est apporté.

Cette observation laisse suggérer que, comme les volailles ont consommé des quantités d'énergie et de protéines constantes, les différences entre leurs caractéristiques nutritionnelles ne reposeront que sur l'équilibre des nutriments qui sont disponibles pour la croissance des poules. Ces différences ont été appréciées, sur la base des valeurs des autres paramètres conventionnels qui servent à mesurer l'efficacité de l'utilisation des aliments par les animaux (Gain moyen quotidien, taux de ponte, qualité de l'œuf et la rentabilité). Les résultats montrent que certains de ces paramètres ont effectivement permis d'opérer une discrimination entre les potentialités nutritionnelles de ces différents régimes examinés. En effet, s'il est vrai que globalement tous les régimes alimentaires utilisés dans cette expérimentation ont provoqué une croissance progressive chez toutes les poules ce qui est 
matérialisé par une augmentation du poids vif, les gains moyens quotidiens (GMQ) obtenus avec les poules soumises aux régimes ont varié en fonction des taux d'incorporation du tourteau d'amande de noix de cajou. La croissance des poules nourries avec les aliments utilisés dans la présente étude pourrait s'expliquer par le fait que ceux-ci ont apporté les protéines indispensables, pour permettre cette croissance (Baiao et Lara, 2005). Cependant, la croissance plus élevée dans le cas de l'incorporation du tourteau d'amandes de noix de cajou s'expliquerait par l'augmentation de la matière grasse dans les régimes contenant le tourteau d'amandes de noix de cajou. Des résultats comparables ont été obtenus par Roth-Maier et al., (1988) chez des poules en incorporant dans leurs régimes 5 à $25 \%$ de tourteaux de canola. En effet, selon Ojewola et al., (2004), les graisses alimentaires améliorent effectivement l'efficacité de l'alimentation des volailles et cette amélioration est attribuée à la concentration énergétique élevée des graisses, tandis que Homer et Schiable (1980) l'ont attribué à la fois à une densité accrue et à une utilisation améliorée de l'énergie des autres constituants alimentaires.

Le taux de ponte moyen des poules soumises au régime R20 était inférieur de 18,21 \% au taux de ponte du régime alimentaire de contrôle Rt. Cela pourrait être lié à une teneur élevée en graisse du régime R20 comparé au régime témoin. En effet, Joly et Bougon (1997) ont montré que les matières grasses élevées dans un régime altèrent les performances des poules pondeuses. L'indice de consommation (IC) n'a pas été influencé par les différents régimes alimentaires jusqu'au taux d'incorporation de $20 \%$ de tourteaux de noix de cajou parce qu'il est lié à la consommation alimentaire et le poids de l'œuf qui également n'ont pas connu de variation.

Le poids de l'œuf est un aspect qualitatif de grande importance économique puisque les œufs sont vendus par classe de poids ou calibre. Les poids moyens des œufs des poules ne variaient pas significativement en fonction du taux d'incorporation de tourteau des noix de cajou ( $0 \%$, ou $10 \%$, ou $15 \%$ ou encore $20 \%$ ). Les poids relevés respectaient également la norme de poids d'œufs défendue par Travel et al. (2010) qui est comprise entre 50 et $70 \mathrm{~g}$ (extrême de 45 à $75 \mathrm{~g}$ ). Des résultats semblables ont été rapportés par Ciurescu (2009) lorsqu'il ajoutait du tourteau de colza dans le régime de poule pondeuse.

La composition globale de l'œuf, les proportions du jaune et du blanc ne présentaient aucune différence significative entre elles, quel que soit le régime alimentaire des poules pondeuses. Les proportions de jaune et de blanc des différents régimes étaient dans la même fourchette que celles des normes qui sont de $60 \%$ et de 30\% respectivement pour le blanc d'œuf et le jaune d'œuf (Gutierrez et al., 1997, Nys et Sauveur 2004).

Le poids moyen des coquilles des œufs des régimes expérimentaux présentait une différence significative. En effet, les tourteaux d'amandes de 
noix de cajou entrainent une dégradation de la qualité de la coquille, ce qui serait lié à une augmentation de la proportion de phosphore dans l'aliment puisque le tourteau de noix de cajou est très riche en phosphore et que selon Bouvarel et al., (2010), il est bien établi qu'un excès de phosphore dans l'aliment altère la qualité de la coquille.

De tous les régimes appliqués, Rt a été le plus coûteux, mais la marge bénéficiaire la plus importante a été obtenue avec le régime R15 suivie de R10. La marge bénéficiaire la moins importante a été obtenue avec le régime R20. Quels que soient les régimes alimentaires, les marges sont positives, indiquant que la conduite des poules pondeuses expérimentées a été bénéfique.

La couleur du jaune est importante pour les consommateurs. Des jaunes bien colorés seraient signe de bonne santé de la poule, mais cette impression est fausse, car la coloration du jaune dépend directement de la consommation en caroténoïdes de la poule. La dégradation de la coloration du jaune de l'œuf serait liée à la quantité des caroténoïdes consommés par la poule, car les animaux ne peuvent pas synthétiser les caroténoïdes. C'est donc uniquement leur alimentation qui détermine la couleur du jaune de leurs œufs (Nys 2000, Bouvarel et al., 2010). Ces auteurs précisent que les principales sources végétales de caroténoïdes utilisées en Europe sont le maïs, le gluten de maïs, la luzerne, les concentrés de luzerne et des extraits de fleurs (soucis, tagètes) ou de plantes (paprika. L'insuffisance de caroténoïdes serait donc liée à la diminution du maïs dans les régimes contenant le tourteau d'amandes de noix de cajou.

Par ailleurs, la marge bénéficiaire ne suffit pas pour témoigner de la rentabilité d'une pratique d'élevage. La rentabilité permet d'apprécier le bénéfice obtenu par rapport à l'investissement initial. Même si le régime R20 dégage un revenu net faible, du point de vue du ratio-bénéfice/coût, l'application de ce régime n'est pas financièrement la moins rentable. Notre étude révèle que la meilleure rentabilité est obtenue avec le régime $\mathrm{R} 15$, par contre le régime témoin Rt a présenté la rentabilité la plus faible. La différence observée au niveau du régime Rt est due au fait que la mise en œuvre a nécessité un investissement élevé en aliment.

En outre, le ratio-bénéfice /coût a été supérieur à 0,5 pour l'ensemble des traitements. Cela implique que pour les quatre régimes, la production a été rentable d'après la théorie de Perrin et al. (1979). Cependant, pour obtenir un rendement supérieur et des avantages économiques, lors de la diffusion de la technologie, il convient de souligner l'importance des bonnes pratiques de production.

\section{Conclusion}

Le tourteau d'amande de noix de cajou a eu un effet satisfaisant sur les paramètres zootechniques, la qualité physique de l'œuf et la rentabilité 
financière de l'exploitation. Les différents résultats expriment dans leur ensemble la bonne valeur nutritionnelle du tourteau d'amandes de noix de cajou qui pourrait être incorporé jusqu'à $20 \%$ dans les régimes alimentaires des poules pondeuses. Ce travail préliminaire sera poursuivi par des études de l'impact sur les caractéristiques nutritionnelles des œufs.

\section{References :}

1. Aduku, A.O. (1993). Tropical feedstuff analysis table. Ahmadu Bello University. Samara, Zaria. Nigeria, P: 1-4.

2. Atteh J.O. et Ologbenla F.D., (1993). Replacement of fishmeal with maggots in broiler diets: Effect on performance and nutrient retention. Nigerian Journal of Animal Production, 1:44-49.

3. Baiao N.C. et Lara L.J.C., (2005). Oil and fat in broiler nutrition. Revista Brasileira de Ciência Avícola, 7: 129-141

4. Bouvarel I., Nys., Panheleux M., Lescoat P. (2010). Comment l'alimentation des poules influences la qualité des œufs. Inra Productions Animales, 23 : 167-182.

5. Ciurescu G. (2009). Efficiency of soybean meal replacement by rapeseed meal and/or canola seeds in commercial layer diets. Archiva Zootechnica, 12: 27-33.

6. Gutierrez M.A., Takahashi H. \& Juneja L.R. (1997). Nutritive evaluation of hen eggs. In Hen eggs, their basic and applied science, Yamanoto T., Juneja L.R., Hatta H., Kim M. eds., CRC Press New York, London, pp.25-35.

7. Hartini S., Choct M., Hinch G. A \& Nolan J., (2003). Effect of diet composition, gut microbial status and fibre forms on cannibalism in layers. A report for the Australian Egg Corporation, pp 30.

8. Homer A. et Schiable J. (1980). Poultry Feeds and Nutrition 2nd Edition, pp: 73.

9. ITAVI (1980). Utilisation du pois dans les aliments de poules pondeuses. 28, rue du Rocher, 75008 Paris, Pp. 22.

10. Jayathilakan K., Khudsia Sultana, K. Radhakrishna, A. S. Bawa (2012). Utilization of byproducts and waste materials from meat, poultry and fish processing industries: a review. Journal of Food Science and Technology, 49(3): 278-293.

11. Joly P. et Bougon M. (1997). Influence du niveau énergétique sur les performances de la pondeuse à œufs roux et évolution de l'ingérée en fonction de l'âge. 2ème journée de la Recherche Avicole- 8-9-10 avril 1997, $2: 115-120$

12. Joly, P. et Loiselet, J. (2005). Niveau énergétiques des aliments pour pondeuses: influence sur les performances et le comportement. 
Sixièmes Journées de la Recherche Avicole, St Malo, 30 et 31 mars 2005, $6: 339-344$.

13. Kouadio K. B., Dougnon M. G., \& Kouakou N. D. V. (2016). Effet de la supplémentation de l'aliment croissance des coquelets (Warren) par du tourteau de Jatropha curcas détoxifié. Journal of Animal \& Plant Sciences, 28(3): 4479-4487.

14. Kouakou N'. D. V., Traoré G. C., Angbo-Kouakou C. E. M., Kouame K. B., Adima A. A., Assidjo N. E., Grongnet J.F., et Kouba M., (2015). Essai préliminaire de production d'oeufs des poules pondeuses (ISA Warren) enrichis en acides gras polyinsaturés oméga 3 avec les graines de Euphorbia heterophylla L. International Journal of Biological and Chemical Sciences 9(4): 1902-1909

15. Larid R., (2012). Valorisation des sous-produits de tomates en vue de leur incorporation dans l'aliment de volaille (Cas des poules pondeuses). Master. Université M'hamed Bougara Boumerdes, Boumerdes, $174 \mathrm{p}$.

16. Lessire M., Hallouis J. M., Chagneau A. M., Besnard J., Travel A., Bouvarel I., Crépon K., Duc G., Dulieu P. (2005). Influence de la teneur en vicine et convicine de la férovole sur les performances de production de la poule pondeuse et la qualité de l'œuf. $6^{\text {ème }}$ journée de la Recherche Avicole, Saint-Malo, France, 6 : 174-178.

17. Mehmet GÜL, M.A. Yörük, T. Aksu, A. Kaya et Ö. Kaynar (2012). The effect of different levels of canola oil on performance, egg shell quality and fatty acid composition of laying hens. International Journal of poultry Science, 11(12): 769-776.

18. Nys Y. \& Sauveur B. (2004). Valeur nutritionnelle des œufs. INRA Production Animale, 17(5) : 385-393.

19. Nys Y., (2000). Dietary carotenoids and egg yolk coloration. A review. Arch. Geflug., 64 :45-54.

20. Odunsi, A.A. (2002). Effect of feeding reject cashew kernel meal on pre and early- laying performance of pullet. Archivos de Zootecnica, $51: 423-429$.

21. Ojewola G.S., Okoye F.C. et Agbakuru I. (2004). Replacement Value of Cashew-nut Meal for Soyabean Meal in Finishing Broiler Chickens. International Journal of Poultry Science, 3 (8): 513-516.

22. Shakeel A. (2010). Effect of supplementing dietary sources of n-3 fatty acids and vitamin a on laying performance, egg quality and immune response in laying hens. Thèse de doctorat. University of Agriculture Faisalabad, Faisalabad, 148 p.

23. Perrin R.K., Winkemann D.L., Moscardi E.R. et Aderson J.R. (1979). Comment établir des conseils aux agriculteurs à partir des données expérimentales. Mexico. CIMMYT (Edicion especial). 38p. 
24. Picard M., Sauveur B., Fenardji F., Angulo I., \& Mongin P. (1993). Ajustements technico-économiques possibles de l'alimentation des volailles dans les pays chauds. INRA Productions animales, 6(2) : 87103.

25. Roth-Maier, A. Dora et M. Kirchgessner (1988). Feeding of 00repeseed to fattening chicken and laying hens. Landwirtsch. Forschung, 41:140-150.

26. Travel A., Nys Y., Lopes E. (2010). Facteurs physiologiques et environnementaux influençant la qualité de l'œuf. In: Numéro Spécial, Qualité de l'œuf. Nys Y. (Ed). Inra Productions Animales, 23 : 155-166. 